\title{
Assessment of Athletic Development in Youth Players - Goal Setting with Normative Data from Basketball
}

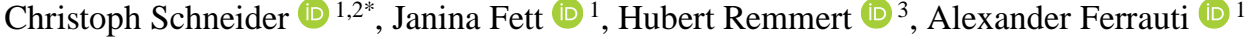 \\ ${ }^{1}$ Department of Training \& Exercise Science, Faculty of Sport Science, Ruhr University Bochum, Bochum, Germany \\ ${ }^{2}$ Department of Cardiology and Angiology, Contilia Heart and Vascular Center, Essen, Germany \\ ${ }^{3}$ Unit of Sport Didactics \& Exercise, Faculty of Sport Science, Ruhr University Bochum, Germany
}

\begin{abstract}
ECSS 2021
INTRODUCTION The nationwide implementation of physical performance test batteries for youth squad players can be valuable for compiling individual physical performance profiles based on age- and gender-specific norm values. This approach is frequently used for optimizing training prescription and thus athletic development. The aim of this study was to introduce a distribution-based approach to derive an effect size scale for assessing athletic development from normative testing data in youth players, which can then be translated to setting performance goals for athletic development.
\end{abstract}

METHODS Secondary analysis of norm values (mixed longitudinal and cross-sectional data [1]). In the age-groups under 12 to under 17, a maximum number of 1,172 and 846 tests were available for male and female basketball squad players, respectively. Biannual testing was conducted as part of a federal research project (20-m sprint, 20$\mathrm{m}$ change of direction sprints with/without basketball, jump \& reach, standing long jump, chest pass, mid-range jump shot, multistage fitness test). An effect size scale was derived from norm values which were available as quintile scores (five categories). Trivial changes were defined as the age-related mean annual performance development which was estimated as the average age-group-to-age-group change for the quintiles. Threshold values for small, medium, and large changes were calculated as average changes that were required to increase performance classification by one, two or three categories, respectively. These thresholds were additionally compared to the default effect size scale commonly used for interpreting standardized mean differences (between-player standard deviation: small 0.2, medium 0.6, large 1.2 [2]).

RESULTS For example, the age-related mean annual development in the jump \& reach for male players was $4 \mathrm{~cm}$ (trivial change). To reach one, two or three higher performance categories, jump height must improve by 8,12 and $15 \mathrm{~cm}$, respectively (i.e., small, medium, large). Compared with the default standardized effect size scale, these quintile-based thresholds were larger.

CONCLUSION The quintile-based analysis presents a simple and practical approach to derive effect size thresholds based on norm values created from regular physical performance testing. These effect size scales can be easily visualized and communicated to players and coaches, as they are typically familiar with percentile-based performance classification of testing data. A limitation of this study was that only norm values in the form of quintile scores were used for analysis. Future research should attempt to model longitudinal datasets while accounting for within- and between-player effects. Furthermore, the choice of appropriate and realistic percentilebased thresholds clearly remains up for debate and requires adequate analysis of original longitudinal data.

REFERENCES 1. Stadtmann (2012) PhD thesis, Ruhr University Bochum. 2. Hopkins (2009) MSSE,41,3-12.

Keywords: Smallest worthwhile change, smallest effect size of interest, normative data, team sports, magnitudebased inferences, youth athletes

Citation: Schneider, C., Fett, J., Remmert, H., \& Ferrauti, A. (2021, April 28). Assessment of Athletic Development in Youth Players - Goal Setting with Normative Data from Basketball. SportRxiv. https://doi.org/10.31236/osf.io/e3tk4

All authors agree to the sharing of this preprint on SportR $\chi i v$.

Twitter: @ChrisSchneiRUB, @FettJanina
SportRxiv is free to access, but not to run. Please consider donating at /www.storkinesiology.org 


\section{Headline}

Using fitness test batteries in youth players can be valuable for compiling individual physical performance profiles and to optimize training prescription and thus athletic development. When implemented at sports federation level, age- and gender-specific norm values are typically available. Such reference data are extremely useful as they enable practitioners to identify individual strength and weaknesses compared to the respective age-group. Although there are various approaches to assessing performance changes and to define cut-off values (1), there is still room for debate and clear guidelines are scares (2).

\section{Aim}

The aim of this report is to present a distribution-based approach to derive an effect size scale for assessing athletic development from normative testing data in youth basketball players, which can then be translated to define explicit performance goals for youth players. Hence, we would like to start a discussion on deriving effect size thresholds, based on available normative data, to help guide the qualitative assessment of youth athletic development and, especially, to support practitioners in reasonable goal setting in youth athletes.

\section{Methods}

The original study and the normative data were previously published as a part of $\mathrm{PhD}$ thesis (3) and booklet in German language (4).

\section{Design.}

Secondary analysis of normative data based on a mixed longitudinal-observational and cross-sectional study.

\section{Athletes.}

Normative data from regular performance testing were available for female and male basketball players from the national D-level and C-level squads. In the age-groups under 12 to under 17 (U12 to U17), a maximum number of 1,172 and 846 tests were available for male and female players, respectively (Table 1).

Table 1 Sample size for male and female player per age-group for which data from at least one performance testing was included in the original analyzes (modified from Tab. 3.2. in 3, p. 43).

\begin{tabular}{lcccccc}
\hline Age-group & U12 & U13 & U14 & U15 & U16 & U17 \\
\hline Male & 86 & 128 & 217 & 387 & 286 & 55 \\
Female & 38 & 73 & 181 & 286 & 187 & 59 \\
\hline
\end{tabular}

\section{Methodology.}

Biannual performance testing was conducted between May 2006 and April 2011 as part of research project Basketball talents which was funded by German Federal Institute of Sport Science (IIA1080703/06-11, IIA1-071613/11-12, IIA1-071614/11-12). With 20 players and a minimum of two test leaders, the testing takes about 2.5 hours. The tests are listed in chronological order in Table 2. Each player performed two trials per test, except for the Multistage Fitness Test. 
Table 2 Performance tests and trial-to-trial reliability.

\begin{tabular}{lc}
\hline Test (unit) & SEM \\
\hline 20-m sprint (s) & 0.05 \\
20-m change of direction sprint (s) & 0.10 \\
20-m change of direction sprint with basketball (s) & 0.19 \\
Jump \& reach (cm) & 2.0 \\
Standing long jump (cm) & 7.4 \\
Chest pass (m) & 0.49 \\
Mid-range jump shot (points) & 2.6 \\
Multistage Fitness Test (level) & \\
\hline SEM: standard error of measurement (standard deviation of differences between trial 1 and 2 divided by $\sqrt{2}$ ). Reliabil- \\
ity data are derived from Table 3.13 of the original PhD thesis (5).
\end{tabular}

\section{Data analysis and descriptive statistics.}

Two distribution-based approaches were used to derive effect size scales describing trivial, small, medium, and large changes.

Trivial changes were defined as the 'average' annual change of performance. To estimate this, we calculated the average age-group-to-age-group change for each quintile. Then, the grand average change was calculated from the mean change for each quintile.

As only normative data were available, the effect size scale was derived based on the originally proposed five performance categories using quintiles as cut-off values (i.e., 20\%-, 40\%-, 60\%-, and 80\%percentile). Therefore, a small, medium, and large change equals the performance change that is needed for a player to reach one, two or three performance categories above the current one in the following age-group (Figure 1).

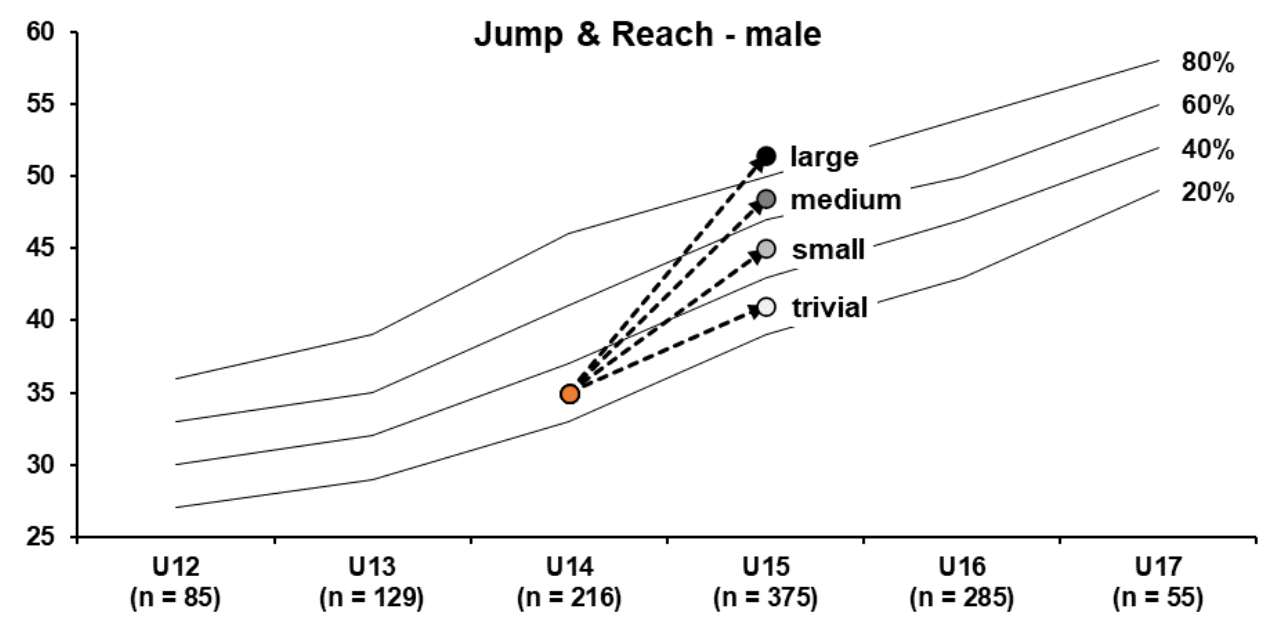

Figure 1 Visualization of the quintile-based effect size approach for a male U15 player with $41 \mathrm{~cm}$ jump height in the jump \& reach test. Trivial: the player remains in the same performance category (i.e., 20-40\%); small, medium, large: improves his performance by one, two, and three performance categories, respectively.

In addition, we calculated effect size thresholds based on modified scale proposed by Hopkins et al. (6) using Cohen's $d$ of 0.2, 0.6, and 1.2 for small, medium, and large effects, respectively. To allow 
comparison to the quintile-based approach described above, these effects magnitudes were added to the average annual change (i.e., small $=$ trivial $+0.2 \mathrm{x}$ between-player SD pooled for all age-groups).

\section{Results}

The effect size scales for the quintile-based approach and the Cohen's $d$-based approach are presented in Table 3.

Table 3 Effect size scales for male and female players using a quintile-based and a Cohen's $d$ based approach.

\begin{tabular}{|c|c|c|c|c|c|c|c|}
\hline \multirow{2}{*}{ Male player } & \multirow[b]{2}{*}{ trivial } & \multicolumn{3}{|c|}{ Quintile-based approach } & \multicolumn{3}{|c|}{ Cohen's $d$ approach } \\
\hline & & small & medium & large & small & medium & large \\
\hline 20-m sprint (s) & -0.11 & -0.20 & -0.30 & -0.40 & -0.15 & -0.22 & -0.32 \\
\hline 20-m change of direction sprint $(\mathrm{s})$ & -0.15 & -0.29 & -0.43 & -0.57 & -0.20 & -0.30 & -0.46 \\
\hline 20-m change of direction sprint with basketball (s) & -0.18 & -0.35 & -0.51 & -0.69 & -0.24 & -0.37 & -0.56 \\
\hline Jump \& reach $(\mathrm{cm})$ & 4 & 8 & 12 & 15 & 6 & 9 & 13 \\
\hline Standing long jump $(\mathrm{cm})$ & 14 & 25 & 36 & 47 & 18 & 26 & 39 \\
\hline Chest pass (m) & 1.2 & 2.1 & 3.0 & 3.9 & 1.6 & 2.2 & 3.1 \\
\hline Mid-range jump shot (points) & 2 & 4 & 7 & 9 & 3 & 5 & 7 \\
\hline Multistage Fitness Test (level) & 0.6 & 1.5 & 2.4 & 3.3 & 0.9 & 1.6 & 2.5 \\
\hline \multicolumn{8}{|l|}{ Female players } \\
\hline 20-m sprint (s) & -0.04 & -0.13 & -0.22 & -0.32 & -0.08 & -0.15 & -0.25 \\
\hline 20-m change of direction sprint (s) & -0.11 & -0.26 & -0.39 & -0.55 & -0.16 & -0.27 & -0.42 \\
\hline 20-m change of direction sprint with basketball (s) & -0.15 & -0.31 & -0.47 & -0.66 & -0.21 & -0.33 & -0.51 \\
\hline Jump \& reach $(\mathrm{cm})$ & 3 & 6 & 9 & 13 & 4 & 6 & 9 \\
\hline Standing long jump $(\mathrm{cm})$ & 8 & 19 & 29 & 39 & 12 & 19 & 29 \\
\hline Chest pass $(\mathrm{m})$ & 0.6 & 1.2 & 1.8 & 2.4 & 0.8 & 1.2 & 1.9 \\
\hline Mid-range jump shot (points) & 2 & 4 & 6 & 8 & 2 & 4 & 6 \\
\hline Multistage Fitness Test (level) & 0.4 & 1.3 & 2.1 & 3.0 & 0.7 & 1.3 & 2.2 \\
\hline
\end{tabular}

\section{Discussion}

The aim of this report was to present a simple approach to derive an effect size scale based on normative testing data, which may then be used for goal setting in youth players. A trivial change was defined as the average annual change in the quantile scores for each test, which thus represents an average performance change that results in a similar relative performance rating in the next age-group. Small, medium, and large changes were calculated as the average change that was required to increase the performance rating by one, two, or three categories, respectively. This arbitrary choice was based on pragmatic reasons, as only the original normative values were available as quintile-scores at the time of analysis. Thus, the choice of appropriate and realistic percentile-based thresholds clearly remains up for debate.

\section{Choosing reasonable effect size thresholds.}

Ideally, effect size thresholds should be chosen specific to the field of application including the test, the measure, and the population of interest (7). More broadly, we may differentiate effect size thresholds that provide qualitative descriptors like trivial, small, medium, and large for different magnitudes of change from thresholds that aim to define a minimum effect size that is required to make a meaningful difference in sports practice. This minimum effect size is also called minimum clinically/practically important difference (MCID/MPID), smallest worthwhile change (SWC) or smallest effect size of interest (SESOI). Typically, the magnitude of a small effect represents such a minimum effect size (7), although there are some circumstances where this may not necessarily be the case and, for example, only medium or large differences would be practically important. The devil is in the detail, and 
these discrepancies may arise due to the various approaches available to define effect sizes and cut-off scores of interest as well as an inconsistent use of those approaches.

In sport science, the use of standardized mean difference (often imprecisely referred to as Cohen's $d$ ) is widespread. In this context, researchers typically interpret the magnitude of the observed effects according to the effect size scale originally proposed by Jacob Cohen (small 0.2, medium 0.5, large 0.7 (8)), or the modified and extended version by Will Hopkins (small 0.2, medium 0.6, large 1.2, very large 2.0, extremely large 4.0 (6)). Although using standardized effect sizes and default thresholds seems straightforward, it was advised to be only used, if no better alternatives are available (8-10). One drawback is, for example, that such distribution-based approaches are strongly influenced by the observed variability in the sample (expressed as standard deviation). Depending on which standard deviation was selected, it may not represent a meaningful measure regarding the effect magnitude. Furthermore, measures of variability can be imprecise in small samples, which are common in sport science research. More importantly, it has to be acknowledged that the original effect size scale by Cohen (8) was empirically derived from the observed effect size distribution in the behavioral and social sciences, and a medium effect being the median value which is "likely visible to the naked eye" (11). So even when using standardized effect sizes seems reasonable, researchers and practitioners are encouraged to determine the specific distribution of effects in each field of application separately (11, 12). An extensive discussion of the different approaches for choosing a minimum target difference can be found elsewhere $(9,10,13,14)$.

\section{Pros and Cons of the quintile-based approach.}

One benefit of the quintile-based approach presented in this report is that it can be easily derived from available normative data in a population of interest. As illustrated in Figure 1, we believe that this approach can also be easily communicated and visualized to coaches and players. The simple logic behind the quintile-based approach is the question: How much do I have to improve my athletic performance within the next year to reach the next higher performance category? Although the choice of the number of percentile-based categories, and therefore the effect size threshold values, is an arbitrary one, it represents a classification approach that is commonly communicated to players and coaches in performance testing reports when classifying athletic performance.

A limitation of the quintile-based approach of this study is that it is based on mixed longitudinalobservational and cross-sectional data. In contrast, the adequate assessment of athletic development seems to demand for a within-subject approach using appropriate longitudinal data and analysis. Only when within-player changes over time are analysed and compared between players, one can evaluate if the here presented effect size scale provides realistic and achievable target values. We cannot clearly rule out if and to which degree the normative values were influenced by selection effects due to the requirement of squad status as an inclusion criterion. For example, players in the higher age-groups may not have been part of the previous age-groups, and some players likely dropped-out. Both effects may in theory influence the progression of the percentiles scores in positive or negative direction. At the moment, we cannot evaluate such potential underlying effects as we are not able to access the original dataset.

\section{Moving forward.}

In case our proposed approach finds some interest and support in the sport science community, we aim to contact the funding agency of the original project as well as the German basketball federation to get access to the original dataset to perform the above-mentioned within-subject analyses. A complementary approach to identifying practically meaningful effect size thresholds could be to evaluate groupdifferences in athletic performance between different selection levels. However, the analyses from the original $\mathrm{PhD}$ thesis (3) suggest that for several tests the mean differences between selection levels were only trivial to small in magnitude and may therefore not always be informative. 
Most importantly, we would like to encourage the sport science community to actively engage in a discussion about the different available approaches to defining meaningful effect sizes. This may ultimately help us to make more informed assessments of the importance of observed effects, and this will also improve our ability to communicate more clearly which effect sizes are actually important to impact sport practice.

\section{Practical applications}

- The effect size thresholds presented in this report may be used to guide the assessment of athletic development and to guide realistic goal setting in youth basketball players.

- The findings may further help starting a discussion among applied sport scientists and practitioners on how to use frequently available normative reference data to derive easy to use benchmarks for evaluation of athletic development.

\section{Limitations}

- The analyses were based on mixed longitudinal-cross-sectional data, although the study's within-subject research question ideally requires a purely longitudinal data sets, which was currently not available.

- The effect size thresholds were based on the arbitrary definition of quantiles as performance cut-offs from the original study authors $(3,4)$.

- Sample size differed (in part substantially) between age-groups and tests.

- We cannot rule out, that training interventions partly influenced the normative data. However, it seems very unlikely that such effects would have been significant for this nationwide and 5year data set.

\section{Conflicts of Interest}

The authors declare that the research was conducted in the absence of any commercial or financial relationships that could be construed as a potential conflict of interest.

\section{Accompanying dataset}

The data are available at the Open Science Framework at (https://osf.io/dgpm3/).

\section{Acknowledgments}

Hubert Remmert and Alexander Ferrauti were part of the research group which realized the original project to develop the test battery.

\section{Twitter}

Follow Christoph Schneider @ChrisSchneiRUB and Janina Fett @ FettJanina

\section{Literature Cited}

1. Buchheit M. Magnitudes matter more than Beetroot Juice. Sport Perform Sci Rep 2018 [cited 2018 Jan 20]:15, v1. Available from: URL: https://sportperfsci.com/magnitudes-matter-morethan-beetroot-juice/.

2. Schneider C, Hanakam F, Wiewelhove T, Döweling A, Kellmann M, Meyer T et al. Heart rate monitoring in team sports: A conceptual framework for contextualizing heart rate measures for training and recovery prescription. Front Physiol 2018; 9.

3. Stadtmann T. Optimierung von Talentselektion und Nachwuchsförderung im Deutschen Basketball Bund aus trainingswissenschaftlicher Sicht [Doctoralthesis]. Ruhr-Universität Bochum, Universitätsbibliothek; 2013. 
4. Ferrauti A, Holst T, Kellmann M, Langenkamp H, Remmert H, Stadtmann T, editors. Basketball Talente: Leitlinien und Empfehlungen auf dem Weg zur Spitze. 1. Auflage. Köln: Sportverlag Strauß; 2015.

5. Stadtmann T. Optimierung von Talentselektion und Nachwuchsförderung im Deutschen Basketball Bund aus trainingswissenschaftlicher Sicht.

6. Hopkins WG, Marshall SW, Batterham AM, Hanin J. Progressive statistics for studies in sports medicine and exercise science. Med Sci Sports Exerc 2009 [cited 2015 Feb 24]; 41(1):3-12.

7. Buchheit M. The numbers will love you back in return-I promise. Int J Sports Physiol Perform 2016; 11(4):551-4.

8. Cohen J. Statistical power analysis for the behavioral sciences. 2. ed. Hillsdale, NJ: Erlbaum; 1988. Available from: URL: http://www.loc.gov/catdir/enhancements/fy0731/88012110-d.html.

9. Cook JA, Julious SA, Sones W, Hampson LV, Hewitt C, Berlin JA et al. DELTA2 guidance on choosing the target difference and undertaking and reporting the sample size calculation for a randomised controlled trial. BMJ 2018; 363:k3750.

10. Caldwell A, Vigotsky AD. A case against default effect sizes in sport and exercise science. PeerJ 2020; 8:e10314.

11. Quintana DS. Statistical considerations for reporting and planning heart rate variability case-control studies. Psychophysiology 2016; 54(3):344-9.

12. Rhea MR. Determining the magnitude of treatment effects in strength training research through the use of the effect size. J Strength Cond Res 2004; 18(4):918-20.

13. Wells G, Beaton D, Shea B, Boers M, Simon L, Strand V et al. Minimal clinically important differences: review of methods. J Rheumatol 2001; 28(2):406-12.

14. Copay AG, Subach BR, Glassman SD, Polly DW, Schuler TC. Understanding the minimum clinically important difference: A review of concepts and methods. Spine J 2007; 7(5):541-6. 